l'affouragement et le "modus vivendi » à l'époque à laquelle commence la paisson, les vicissitudes du climat, les caractéristiques individuelles du cheptel élevé, ete., mettent davantage à l'épreuve et pour une durée plus longue la capacité de résistance.

L'explication classique pour la prévention de la modification de la composition du lait, ou mieux, son atténuation, offre d'ellemême les procédés généralement connus, assurant les phénomènes vitaux normaux.

Les données de l'expérience confirment que là où, en ce qui concerne le elimat, la quantité et la qualité du fourrage, la transition était graduelle au point de vue physique, les éléments examinés du lait n'ont atteint que temporairement et dans une faible mesure, un taux supérieur à celui de la valeur normale.

Etant donné que la teneur en extrait see dégraissé n'a pas paru constante, l'hypothèse que son examen pourrait prouver ou démentir la falsification ne s'est done pas vérifiée.

(Travail de la Faculté des sciences économiques de l'Institut zootechnique de Budapest. Directeur : Prof. J. SoHandL.)

\title{
CONSIDÉRATIONS SUR L'INFLUENCE DU PASSAGE DANS LE LAIT DE QUELQUES PRINCIPES D'ORIGINE MINÉRALE OU VÉGÉTALE
}

\author{
par M. LAGRANGE-FRANCÈS
}

Docteur en pharmacie.

Il est bien connu que nombre de médicaments passent dans le lait. Sigalas et Dupoux, notamment, ont montré que le lait des nourrices soumises à un traitement hydrargyrique contient du mercure; Dervieux, Szumlanski et Desvillees ont montré la présence d'alcool dans le lait d'une jeune aleoolique et le passage de ce composé dans les organes de son nourrisson de 4 mois, mort de broncho-pneumonie.

D'autre part, PoroHer a écrit :

"Si l'on tient pour fondé le passage des substances médicamenteuses dans le lait, la question mérite qu'on s'y arrête et cela pour deux raisons :

$1^{\circ} \mathrm{Qu}$ 'on veuille préparer des laits dits médicamenteux,

$2^{\circ}$ Que l'on tienne, dans un esprit cette fois tout opposé, à interdire, comme le fait le règlement de plusieurs villes allemandes, la vente du lait d'animaux ayant absorbé certains produits dont, à tort ou à raison, on redoute le passage dans le lait. "

D'autre part, beaucoup d'auteurs ont signalé les modifications organoleptiques du lait d'animaux a yant absorbé certains végétaux. 
Voici quelques-unes de ces observations, sommairement résumées :

Plantes donnant une couleur particulière au lait.

a) Rouge pâle .... le caille-lait, ou grateron (gallium verum); là garance (rubia tinctoria).

b) Jaune pâle..... la carotte (daucus carota);

le safran (crocus sativus);

le rapontic (rheum raponticum) ;

le tourteau de sésame;

les tourteaux de crucifères.

c) Bleu ......... la bourrache bâtarde (anchusa officinalis);

le jonc fleuri (butomus umbellatus);

le mélampyre, ou queue-de-renard (melampyrum arvense) ;

la mercuriale (mercuriatis perennis);

la traînasse (polygonum aviculare) ;

le cocriste, crête-de-coq (rhinantus major); la prèle (equisetum arvense).

Toutes ces couleurs n'apparaissent que sous l'acidification spontanée du lait, ce qui fait penser que les matières colorantes en question sont éliminées à l'état de chromogènes.

Plantes qui donnent au lait un goût particulier.

L'ail (allium verum)...... )

La ciboule ............ (saveur âcre et brûlante).

Le poireau $\ldots \ldots \ldots \ldots . . .$. .

L'absinthe (artemisia absintium) (saveur d'absinthe particulière).

Les navets et les raves (brassica rapa et brassica napus) (odeur sulfurée).

L'euphorbe, ou rhubarbe des pauvres (euphorbia siparisias) (odeur et saveur désagréables, âcre).

La gratiole (gratiola officinalis) (sa veur âcre).

L'ellébore (helleborus niger) (saveur âcre).

La camomille commune (matricaria chamomilla) (odeur et saveur fortes).

La chicorée (cichorium intybus) (goût amer, propriété laxative du lait).

Le mais (zea mays) (odeur et saveur fades).

Le trèfle (trifolium pratense) (odeur et goût très agréables).

Les labiées (odeur et goût agréables).

Le fenouil (feniculum dulce) (odeur et saveur anisées).

Le marron d'Inde, l'artichaut, le genêt, le sureau, les pommes de terre germées donnent un goût amer et désagréable. 
Le tourteau de colza donne un goût et une odeur désagréables, plus perceptibles dans la crème.

Les collets de betterave, dont les feuilles sont souvent en décomposition, par suite de la culture du bacillus fluorescens, donnent au lait un goût et une odeur de pourri.

Les drèches donnent un goût fade et une odeur désagréable (docteur RoskaM).

Les tourteaux d'arachides rances donnent au lait un goût de rance.

La paille moisie et les navets lui donnent un goût amer.

Plantes qui facilitent la coagulation du lait.

Le kebracho (aspidosperma quebracho).

Le papayer (carica papaya).

La cirse des champs (circium arvense).

L'oseille (oxalis acetallosa).

La chardonnette (cynara cardunculus).

Le figuier (ficus carica). (On obtient a vec les feuilles une coagulation plus rapide $q u^{\prime} a v e c$ le fruit.)

Le poivre (piper nigrum).

Le chêne (quercus infectoria).

La patience (rhumex patientia).

Plantes qui empêchent la coagulation du lait.

Le cochléria (cochlearia officinalis). (A ce propos, il existe une curieuse observation de STonckLIN : un lait rosissait chaque fois qu'on faisait cuire dans un récipient en fer. Il fut montré que la vache dont provenait ce lait absorbait une notable quantité de erucifères riches en sénevols.)

La sanicule (sanicula europea) (Journal du Muséum de Berlin, 1880).

Nous avons d'ailleurs contrôlé nous-mêmes (1) l'exactitude de ces faits pour : la garance, la carotte, le rapontic, la bourrache, la mercuriale, la prèle, l'ail et le poireau, le navet et les raves, la chicorée, le maiis, le fenouil, le genêt, les collets de betteraves, le tourteau d'arachides, la chardonnette, le figuier, la patience.

\section{HUILE DE FOIE DE MORUE}

Partant des principes que nous venons de rappeler, nous avons étudié les modifications que peut faire subir l'absorption d'huile de foie de morue, MM. LABAT et Chelle nous ayant signalé qu'ils avaient souvent observé que le beurre extrait du lait de nourrices a yant ingéréce médicament, présentait toujours une odeur prononcée de poisson.

(1) A. Lagranger : Etude analytique des laits de la vallée de la Dronne. Thèse Doct.. Pharm., Bordeaux, 1933. 
Le 11 décembre 1931, nous avons soumis au traitement par l'huile de foie de morue, une vache hollandaise de 8 ans, ayant vêlé au mois de juillet dernier, produisant 11 litres de lait par 24 heures, recevant comme nourriture habituelle du foin, des pommes de terre, du son et le pacage le soir.

Avant le traitement, nous avions comme constituants :

\begin{tabular}{|c|c|}
\hline & 1,8 \\
\hline Extrait sec & 131,20 \\
\hline Extrait débeurré .. & 93 \\
\hline Matières grasses..... & 38,20 \\
\hline Lactose ........... & 49 \\
\hline Caséine $\ldots \ldots \ldots \ldots \ldots \ldots \ldots \ldots$ & 31,3 \\
\hline Chlorures...$\ldots \ldots \ldots \ldots \ldots \ldots$ & 1,65 \\
\hline Cendres $\ldots \ldots \ldots \ldots \ldots \ldots \ldots \ldots \ldots$ & 6,85 \\
\hline C. M. S. $\quad \ldots \ldots \ldots \ldots \ldots \ldots$ & 72,92 \\
\hline
\end{tabular}

Après 8 jours de traitement à l'huile de foie de morue, avec 100 grammes tous les matins, nous a vons constaté qu'en débouchant la bouteille, une odeur d'huile de foie de morue se dégageait, pas de changement de couleur, une saveur légère d'huile de foie de morue, surtout dans la crème; l'analyse de ce lait nous a donné :
1,75
Extrait sec
131,75
Extrait débeurré
92,75
Matières grasses ............. $\quad 39$
Lactose .................. 50
Caséine .................... 31
Chlorures $\ldots \ldots \ldots \ldots \ldots \ldots \ldots \ldots \quad 1,55$
Cendres.................. 6,80
C. M. S. $\ldots \ldots \ldots \ldots \ldots \ldots \ldots \ldots \ldots \ldots \ldots \ldots \ldots \ldots$ 72,71

$\mathrm{Au}$ bout de 15 jours de traitement, nous avons eu :

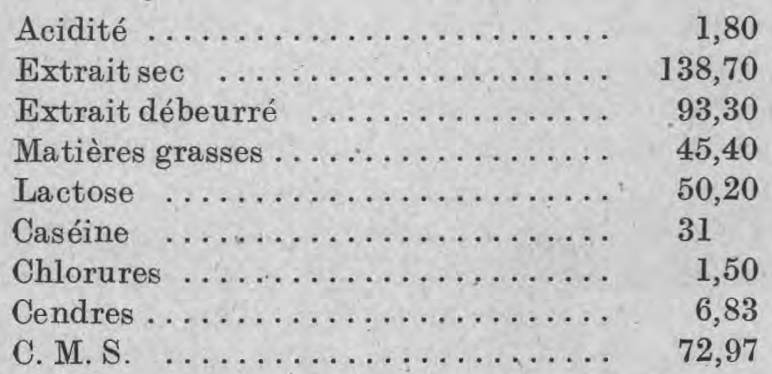

Nous avons refait cette expérience sur différentes vaches en Dordogne, Charente et Charente-Inférieure, toujours au bout de 15 jours; il y avait augmentation de la matière grasse.

A ce moment, nous avons observé que le beurre provenant du 
lait des vaches ainsi traitées était sensiblement plus coloré qu'avant le début de l'expérience.

Pour chiffrer en quelque sorte cette augmentation de teinte, nous a vons procédé comme suit. On extrait du lait examiné suffisamment de beurre pour pouvoir en peser 5 grammes. On fait dissoudre ce poids dans $10 \mathrm{~cm}^{3}$ de réactif d'Adam et on verse la solution obtenue dans un tube à essai, qu'on bouche hermétiquement, pour éviter l'éva poration. D'autre part, on prend une solution bien titrée à $1 \mathrm{gr}$. par litre de chromate de potassium et on la dilue jusqu'à ee que l'on obtienne une teinte égale à celle de la solution de beurre dans le solvant éthéro-alcoolique. On peut ainsi estimer numériquement la teinte du beurre.

Par exemple, l'expérience citée plus haut nous a donné les chiffres suivants :

\begin{tabular}{|c|c|}
\hline Temps & $\begin{array}{c}\text { Teinte, la solution } \\
\text { butyrique exprimée } \\
\text { en chromate de } \\
\text { potassium } \\
\text { pour mille }\end{array}$ \\
\hline Avant expérience. & $\ldots \quad 0$ gr. 035 \\
\hline 8 jours a près...... & $0 \mathrm{gr} .050$ \\
\hline 15 jours a près $\ldots .$. & 0 gr. 070 \\
\hline
\end{tabular}

Il s'ensuit done qu'il y a passage manifeste dans la matière grasse du lait de certains principes provenant de l'huile de foie de morue.

Or, d'après les travaux de Fernando Sanchez Gerono (étude des vitamines dans les huiles), il semblerait que la vache soumise au traitement de l'huile de foie de morue élimine par son lait une plus grande quantité de vitamine $\mathrm{A}$, qui serait fixée dans la matière grasse.

Il est vraisemblable que l'augmentation de la coloration du beurre est liée à cette richesse en vitamine $\mathrm{A}$ (carotène). En utilisant la comparaison de la teinte de cette matière grasse avec celle d'une solution titrée de chromate de potassium, comme nous l'avons indiqué précédemment, peut-être, aurait-on un moyen simple et rapide de classer les différents laits en fonction de leur richesse en vitamine A.

En tout cas, une mère a consenti de donner à son enfant, régulièrement, pendant deux mois, un litre de lait provenant d'une vache traitée à l'huile de foie de morue. Cet enfant, âgé de 14 mois, n'a montré ni dégoût, ni troubles intestinaux. $\mathrm{Il}$ a grandi et augmenté de poids de façon très normale.

Peut-être, des expériences analogues à celle-ci mériteraient-elles d'être multipliées et en tirerait-on des conclusions intéressantes concernant l'alimentation des jeunes enfants. 
Nota. - Porcher a signalé que le lactate de cuivre ajouté à l'alimentation de la vache communique au beurre une odeur rappelant eelle de l'huile de poisson, mais encore, plus désagréable. Nous avons constaté l'exactitude de cette affirmation.

\section{CAROTTES ROUGES}

Il y a une douzaine d'années, STEENBOCK avait remarqué des relations entre la vitamine $\mathrm{A}$ et les carotinoïdes. Actuellement, on sait que cette vitamine est très voisine, sinon identique au carotène de Tanret, ou, plus exactement, le carotène serait la provitamine A.

Les carottes blanches, mais surtout les carottes rouges, nous ont permis d'observer sur deux hollandaises (CHASTENET) des faits intéressants durant les mois de décembre 1931 et janvier 1932 :

Pendant 15 jours, d'abord, nous avons donné le matin $1 \mathrm{~kg}$. 500 de carottes rouges à ces deux vaches : aucune variation, ni changement de teinte dans ce lait; l'une produisait 12 litres et l'autre 9 litres par jour. A près 1 mois de ce traitement, où nous avons élevé la ration à $3 \mathrm{~kg}$. par jour, nous a vons constaté une légère diminution dans le rendement ( 101.500 et 8 litres), sans pouvoir affirmer si cela était dû à l'absorption des carottes ou au brusque abaissement de la température à cette époque de l'année. Toujours est-il que, de l'avis des consommateurs, le lait "s'était amélioré et était devenu plus crémeux ». Il y avait augmentation de beurre de 33 gr. pour mille à $41 \mathrm{gr}$. La coloration du lait avait changé et était jaune pâle (le beurre, également, avait une coloration jaune, plus foncé).

Or, d'après Srmonnet, "le régime alimentaire des femelles laitières joue un rôle essentiel dans la valeur du lait qu'elles produisent; la valeur du lait en temps que vecteur des facteurs accessoires, montre qu'il est surtout riche en facteur A, mais qu'il n'est que médiocrement pourvu en facteurs B et $\mathrm{C}$ ).

D'a près les travaux de Jean VERNE, nous savons que :

"Les carottes rouges riches en carotinoïdes apporteraient la provitamine $\mathrm{A}$ (carotène), non active par elle-même, mais qui donnerait naissance au corps actif. La croissance de certains animaux carencés soumis à cette alimentation aurait augmenté. 》

E. Lesné, médecin de l'hôpital Trousseau, et H. Vagliaño, chef de clinique de la faculté d'Athènes, ont pu eonstater que la glande mammaire n'a pas la faculté de faire la synthèse des vitamines A, B, C, D. Il faut que ces substances soient a pportées à l'organisme maternel par les aliments. Le lait ne les renferme qu'à cette condition. La glande mammaire élimine les vitamines A, B, C, comme le rein sécrète l'urée et le chlorure de sodium.

Il existe, d'a près ces auteurs, incontesta blement un rapport entre la quantité de vitamines ingérées par la vache et la quantité de vitamines éliminées par la glande mammaire. 
Or, en rapprochant ces remarques des constatations que nous a vons faites a près administration à la vache d'huile de foie de morue (coloration du lait et du beurre augmentée, poids de matière grasse plus élevé), et a près ingestion de carottes (coloration du beurre plus intense et goût plus agréable), nous avons pensé qu'il y aurait intérêt, pour la santé des enfants, pour leur développement et leur croissance, à donner des laits riches en vitamines.

L'expérience semble confirmer nos observations. Nous a vons fait a bsorber quotidiennement à des vaches, a vec leur ration habituelle, $3 \mathrm{~kg}$. de carottes et une dose de 150 à $200 \mathrm{gr}$. d'huile de foie de morue. Une jeune mère, dont le mari est domestique au Fieu, ayant sevré son enfant, a donné, pendant l'hiver 1931-1932, ce lait supposé ainsi survitaminé. Nous avons constaté que la santé de l'enfant a été parfaite pendant toute cette période ; sa taille et son poids ont normalement augmenté.

Nous croyons devoir signaler ces observations pour le plus grand intérêt de la théra peutique infantile : la pratique des substitutions alimentaires des grandes femelles laitières deviendrait une nécessité.

\title{
L'ACIDE PICRIQUE DANS LE LAIT. SA CARACTÉRISATION
}

\author{
par M. LAGRANGE-FRANCÈS
}

Docteur en pharmacie.

En opérant sur plusieurs animaux, nous avons constaté que lorsqu'on administre à une vache des doses eroissantes d'acide picrique, il arrive un stade où le lait prend un aspect jaunâtre et garde cette teinte-limite quelle que soit la quantité d'acide picrique ingérée en excès.

L'apparence du lait est ainsi très favorable; sa couleur peut en effet laisser supposer qu'il est très riche en beurre:

Son odeur n'est pas modifiée. Il ne présente aucune saveur anormale, contrairement à ce que l'on aurait pu croire. Un enfant l'accepte très volontiers. L'acidité n'est pas augmentée.

On ne note aucune variation appréciable dans les éléments normaux du lait.

Or, si un fraudeur ajoute de l'eau à un lait normal, ce lait prend une teinte bleutée caractéristique. Avec un lait picriqué, au contraire, l'addition d'eau passe inaperçue en apparence : l'aspect du lait reste favorable, et le consommateur non averti ne peut soupçonner la fraude à simple vue.

Bien qu'à notre connaissance, cette fraude n'ait jamais été pratiquée, il nous a paru intéressant d'étudier comment on pourrait 